Background The major role of an ethics committee is to protect participants from harm through participating in a health research study. This includes investigating participant complaints to ensure that their wellbeing is being upheld and queries resolved satisfactorily. In this case, one clinical trial participant passed away and a report implying that her death was caused by trial medication received during participation was published by WEMOS foundation. This necessitated the National Ethics Committee (NEC) in Zimbabwe to investigate the case to understand and come up with resolutions.

Methods A case study design was used to investigate the case. Interviews with 5 conveniently selected study staff based on their involvement and roles in the study were conducted. Review of participant study records, the protocol, the WEMOS report, Serious Adverse Event reports and Data Safety and Monitoring Board reports were conducted. The investigation was used to determine which ethical principles applied and whether they were adhered to or not in the handling of the participant by the research staff.

Results Results indicated that the research team adhered to the necessary ethical principles enshrined in the major ethical codes and local Zimbabwean research ethics regulations for the conduct of clinical trials. Investigation showed that the report was mainly based on incomplete information and contradicted the actual events at the study site. There was also no record of the participant's complaint with NEC in the complaint register. Appropriate standard of care was given to the participant.

Conclusions The NEC continues to protect the rights of clinical trial participants by investigating complaints against study teams as their wellbeing is of primary importance. Researchers are being encouraged to adhere to best practises in conducting human participant researches. The media should also be engaged actively so that reporting is accurate to prevent incorrect information being relayed to the public.

\section{PA-085 ETHICAL CONSIDERATIONS IN THE HANDLING OF A COMPLAINT REPORT AGAINST A STUDY TEAM: CASE OF A CLINICAL TRIAL (EARNEST) PARTICIPANT}

Muchineripi Kanengoni, Sithembile Ruzario, Paul Ndebele, Melody Shana, Fadzai Tarumbiswa, Rosemary Musesengwa, Rutendo Gutsire. MRCZ, Zimbabwe 